\title{
Antibiotic-Producing Beneficial Bacteria in the Gut of the Burying Beetle Nicrophorus vespilloides
}

\section{OPEN ACCESS}

Edited by:

Ezequiel Miguel Marzinelli,

University of Sydney, Australia

Reviewed by:

Om Vir Singh,

TSG Consulting, United States

Olaf Tyc,

Netherlands Institute of Ecology

(NIOO-KNAW), Netherlands

*Correspondence:

Andreas Vilcinskas

Andreas.Vilcinskas@

agrar.uni-giessen.de;

Andreas.Vilcinskas@ime.fraunhofer.de

Specialty section:

This article was submitted to

Microbial Symbioses,

a section of the journal

Frontiers in Microbiology

Received: 12 March 2019

Accepted: 08 May 2019

Published: 31 May 2019

Citation:

Heise P, Liu Y, Degenkolb T, Vogel H, Schäberle TF and

Vilcinskas A (2019)

Antibiotic-Producing Beneficial Bacteria in the Gut of the Burying

Beetle Nicrophorus vespilloides.

Front. Microbiol. 10:1178.

doi: 10.3389/fmicb.2019.01178

\section{Philipp Heise', Yang Liu'2, Thomas Degenkolb², Heiko Vogel ${ }^{3}$, Till F. Schäberle 1,2,4 and Andreas Vilcinskas ${ }^{1,2 *}$}

\begin{abstract}
1 Department of Bioresources, Fraunhofer Institute for Molecular Biology and Applied Ecology, Giessen, Germany, ${ }^{2}$ Institute for Insect Biotechnology, Justus Liebig University Giessen, Giessen, Germany, ${ }^{3}$ Department of Entomology, Max Planck Institute for Chemical Ecology, Jena, Germany, ${ }^{4}$ German Center for Infection Research (DZIF), Partner Site Giessen-Marburg-Langen, Giessen, Germany
\end{abstract}

The increasing prevalence of antibiotic-resistant human pathogens is a growing public concern and there is intense pressure to identify new antibacterial compounds that can be developed into antibiotics with novel mode of action. Evolutionary theory predicts that insects that have evolved to occupy sophisticated ecological niches by feeding and reproducing on carcasses will depend on their gut microbiome to prevent colonization by invading pathogens taken up with the diet. This inspired our hypothesis that the complex interactions between the core microbiome and the more flexible microbial communities dependent on the environment may promote the outsourcing of antibiotic synthesis to beneficial microbes. We tested this hypothesis by cultivating and characterizing bacteria isolated from the gut of the burying beetle Nicrophorus vespilloides, which feeds and reproduces on small vertebrate carcasses buried in the soil to avoid competitors such as fly maggots. The extracts of isolated bacteria were screened for activity against human pathogens such as Escherichia coli, Pseudomonas aeruginosa, Staphylococcus aureus, and Candida albicans. More than 400 strains were isolated, among which the crude extract of Serratia marcescens 2MH3-2 displayed promising activity against Staphylococcus aureus. Bioactivity-guided fractionation enabled purification of the primary antimicrobial compound of the extract. By LC-MS and NMR experiments, it was identified as serrawettin $W 2\left(\mathrm{C}_{38} \mathrm{H}_{61} \mathrm{~N}_{5} \mathrm{O}_{9}\right)$, the antibacterial and nematostatic activity of which was corroborated in our study. We postulate that this antibiotic could contribute to the control of both bacteria and phoretic nematodes in the gut, which compete for food when transferred to the carcass. Our study shows that the gut microbiome of $N$. vespilloides is a promising resource for the screening of antibiotic-producing bacteria.

Keywords: symbiosis, gut microbiome, serrawettin W2, Nicrophorus vespilloides, burying beetle, nematodes, bioactivity-guided screening, natural products

\section{INTRODUCTION}

The spread of antibiotic-resistant pathogens is a global challenge that causes up to 700,000 deaths per year, and mortality rates are expected to rise if novel antibiotics, particularly targeting Gramnegative bacteria, are not developed in the near future (Stern et al., 2017). New antimicrobials have been sought by screening industrial chemical libraries, but this has failed to provide new 
candidates. However, the vast majority of available antibiotics are natural products or derivatives thereof. Consequently, natural product research requires new strategies to revitalize declining natural product screening initiatives (Rossiter et al., 2017; Wright, 2017). One option is to expand the microbial strains used for natural product screening beyond Actinomyces and Streptomyces to include strains from microbial phyla that are neglected (Lasken and McLean, 2014). Another recent initiative focuses on the human gut microbiome as a resource for natural products (Wilson et al., 2017). This is based on the fact that a healthy gut microbiome can prevent invading pathogens colonizing the gastrointestinal tract, a phenomenon known as colonization resistance (Kim et al., 2017). Therefore, we explored the premise that particular insects, which are adapted to feed and reproduce on heavily-contaminated and microbe-rich sources, should harbor bacteria that produce antimicrobial compounds. Insects are particularly good candidates for discovering novel antibiotics and antibiotic producers due to their ancient mutualistic relationship with several microorganisms, which protect insect hosts against pathogens and parasites, thus allowing insects to colonize harsh environments, protect and preserve food resources, and prevent infection in their offspring (Flórez et al., 2017).

Burying beetles such as Nicrophorus vespilloides occupy a unique ecological niche by feeding and reproducing on carcasses, a nutrient-rich but ephemeral source that is subject to intense microbial competition (Vogel et al., 2017; Miller and Cotter, 2018). They display parental care by preparing the carcass as a diet for their offspring (Pukowski, 1933; Jacobs et al., 2016). This necessarily encompasses the regulation of the carcass-associated microbiome, which is used to transmit a core microbial community to their offspring (Shukla et al., 2018a; Wang and Rozen, 2018). Feeding and reproducing on carcasses requires adaptations to cope with both soilborne and cadaver-associated microbes, which can harm also the eggs of $N$. vespilloides laid in close proximity to the decomposing carcass (Jacobs et al., 2014). N. vespilloides beetles are protected by a diverse spectrum of antimicrobial peptides, which are expressed in a context-dependent manner (Vogel et al., 2011; Jacobs et al., 2016) but they also sanitize the carcasses with oral and anal secretions consisting of at least 34 different small molecules with antimicrobial activity (Degenkolb et al., 2011). Four of these compounds were recently found to be produced by symbiotic Yarrowia-like yeasts that colonize the beetles' hindgut (Vogel et al., 2017). This finding inspired our hypothesis that the $N$. vespilloides gut microbiome may also harbor beneficial bacteria producing low-molecular-weight bioactive compounds, as reported for other insect hosts (Kroiss et al., 2010; Flórez et al., 2017; Engl et al., 2018). However, competing organisms that live on the carcasses are not restricted to the kingdoms of bacteria and fungi, respectively (Shukla et al., 2018b), but also include phoretic nematodes that use burying beetles as a vehicle for transmission to carcasses used as a diet and for reproduction (Richter, 1993). A recent transcriptomic analysis of the carcass attended by $N$. vespilloides confirmed the transfer of living nematodes that are generally harmless but act as competitors for carcass-derived nutrients (Vogel et al., 2017). In contrast, other phoretic nematodes associated with this burying beetle (such as Rhabditoides regina) are parasites, with detrimental effects against the feeding larvae (Wang and Rozen, 2019).

To test our hypothesis that bacterial symbionts in the gut of $N$. vespilloides may provide protection against both competing microbes and phoretic nematodes, we isolated, cultured and screened bacteria from the beetle's gut microbiome for the ability to inhibit (i) obligate and facultative human pathogens such as Escherichia coli, Pseudomonas aeruginosa, Staphylococcus aureus, Candida albicans and Mycobacterium smegmatis; and (ii) the model nematode Caenorhabditis elegans. We screened more than 400 isolates and culture extracts to identify symbiotic bacteria with the strongest activity against pathogens and nematodes, and used a combination of analytical techniques in an attempt to identify active compounds. Our findings allowed us to propose an ecological role for antimicrobial and nematicidal molecules in the novel context of $N$. vespilloides parental care.

\section{MATERIALS AND METHODS}

\section{Insect Rearing and Treatment}

We used $N$. vespilloides beetles either caught in the wild during the summer in Giessen (Germany) or bred in captivity by H. Vogel (Max Planck Institute for Chemical Ecology, Jena, Germany). The wild beetles were caught by placing a dead mouse (Mus musculus) on soil in a modified container with a lid pierced to create a hole $\sim 3 \mathrm{~cm}$ in diameter. The container was buried with the lid at ground level and was checked daily. The captured beetles were first cooled to reduce movement and then washed sequentially in $1 \% \mathrm{H}_{2} \mathrm{O}_{2}$, sterile water, $70 \%$ ethanol and again in water for $1 \mathrm{~min}$ each to reduce surface contamination risks. The surface-sterilized beetles were dissected and the entire gut (from foregut to rectum) was removed. Gut sections were macerated in sterile phosphate-buffered saline (PBS) using a sterile pipette tip, and each preparation was serially diluted. Fifty $\mu$ l of the dilutions $10^{-3}$ to $10^{-6}$ were plated on brain heart infusion (BHI) medium (Carl Roth, Karlsruhe, Germany), water agar and Trypticase soy yeast extract (TSBYE; Sigma-Aldrich, Munich, Germany). Each medium was prepared with $1.5 \%$ agar. Additionally, the same media have also been used in parallel, supplemented with ampicillin and kanamycin (see next paragraph).

\section{Bacterial Cultivation and Identification}

Microbes were cultivated on BHI agar or TSBYE agar for 14 days or on water agar for up to 3 weeks. Additionally, antibiotic-resistant cultures were selected in parallel on media supplemented with $0.05 \mathrm{mg} / \mathrm{ml}$ ampicillin and $0.025 \mathrm{mg} / \mathrm{ml}$ kanamycin. All cultures were incubated at $28^{\circ} \mathrm{C}$. If growth was too rapid for colony selection, we reduced the temperature to $21^{\circ} \mathrm{C}$ (room temperature) and/or reduced the nutrient yield to $10 \%$ (e.g., for the genus Proteus). Colonies were visually selected, picked and streaked onto fresh agar until no contamination was detected by eye or microscopy. Isolates were then kept in Roti-Store cryovials (Carl Roth) at $-80^{\circ} \mathrm{C}$. 
Isolates from the $N$. vespilloides gut were identified by $16 S$ rDNA gene amplification using a C1000 Thermal Cycler (BioRad, Hercules, CA, United States) followed by Sanger sequencing (Eurofins, Ebersberg, Germany). We used the standard primer set 27F ( $5^{\prime}$-AGA GTT TGA TCM TGG CTC AG-3') and 1492R (5'ACC TTG TTA CGA CTT-3') (Lane, 1991). Each amplification was based either on a standard colony PCR or was achieved by first lysing the cells in $0.2 \%$ SDS. For the standard colony PCR, cell material was picked using a pipette tip and mixed with $30 \mu \mathrm{l}$ double-distilled water before heating the cell suspension to $95^{\circ} \mathrm{C}$ for $5 \mathrm{~min}$. We used 1-3 $\mathrm{\mu l}$ of each cell lysate as a PCR template.

The PCR comprised 32 cycles of denaturation $\left(95^{\circ} \mathrm{C}, 5 \mathrm{~min}\right)$, annealing $\left(56^{\circ} \mathrm{C}, 30 \mathrm{~s}\right)$ and extension $\left(72^{\circ} \mathrm{C}, 1 \mathrm{~min}\right)$ followed by a final elongation at $72^{\circ} \mathrm{C}$ for a further $1 \mathrm{~min}$ before storage at $4^{\circ} \mathrm{C}$. The PCR products were separated by $1.5 \%$ agarose gel electrophoresis at $110 \mathrm{~V}$ for $40 \mathrm{~min}$, and product bands were isolated using a Gene Jet gel extraction kit (Qiagen, Hilden, Germany). After Sanger sequencing as above, the sequences were identified using the NCBI Basic Local Alignment Search Tool (BLAST).

MEGA 7 (molecular evolutionary genetics analysis) was used to align the obtained $16 S$ gene sequences of the Serratia isolates to $16 S$ sequences of Serratia type strains from the NCBI database. From this alignment, a maximum-likelihood phylogenetic tree was generated and circularized in order to improve its visibility.

\section{Chemical Extraction and Bioassay}

We established three 100-ml cultures in 300-ml Erlenmeyer flasks from selected isolates based on $16 S$ gene sequences. Fermentation of approximately 130 bacterial isolates was carried out at $28^{\circ} \mathrm{C}$, shaking at $140 \mathrm{rpm}$ for 9 days with three harvesting points (days 1, 6, and 9). The cells were harvested directly into liquid nitrogen and lyophilized after shell freezing. Extracts were prepared in $50 \mathrm{ml}$ ethyl acetate with $15 \mathrm{~min}$ ultrasonication to break up the cells. After filtration, a second extract was prepared as described above, using methanol instead of ethyl acetate. Both crude extracts were then concentrated 100-fold and stored at $4^{\circ} \mathrm{C}$.

The bioactivity tests were set up against clinically relevant strains and tested in duplicate using 10, 1, 0.5, and $0.25 \mu \mathrm{l}$ of each crude extract in 96-well plates to ensure a high throughput. For Staphylococcus aureus, E. coli, and C. albicans, $100 \mu \mathrm{l}$ of cation-adjusted Müller-Hinton broth was inoculated with $\sim 5 \times 10^{5}$ cells $/ \mathrm{ml}$ and incubated at $37^{\circ} \mathrm{C}$ for $18 \mathrm{~h}$, shaking at $180 \mathrm{rpm}$. Growth inhibition was measured against both positive and negative controls at $600 \mathrm{~nm}$ (gentamicin serial dilution/fluconazole) using a plate reader.

M. smegmatis was grown for $48 \mathrm{~h}$ at $37^{\circ} \mathrm{C}$ in $\mathrm{BHI}$ medium supplemented with $1 \%$ Tween-80. Afterward, the cell suspension was diluted to $\sim 1 \times 10^{5}$ cells $/ \mathrm{ml}$ in cation-adjusted MüllerHinton broth, and $100 \mu \mathrm{l}$ of the diluted suspension was dispensed into white 96 -well flat-bottom plates. The plates were incubated for $48 \mathrm{~h}$ at $37^{\circ} \mathrm{C}$, shaking at $180 \mathrm{rpm}$. Cell viability was determined using the BacTiter-Glo ${ }^{\text {TM }}$ assay (Promega, Mannheim, Germany) and a LUMIstar OPTIMA microplate luminometer (BMG Labtech, Ortenberg, Germany) for the readout (Dardic et al., 2017).

\section{Antimicrobial Screening}

The following strains were used to determine antimicrobial activity: E. coli ATCC 25922, P. aeruginosa ATCC 27853, methicillin-sensitive Staphylococcus aureus ATCC 25923 (MSSA), C. albicans (in-house strain) and M. smegmatis ATCC 607. The minimal inhibitory concentration (MIC) was also evaluated against Bacillus subtilis DSM 10, methicillin-resistant Staphylococcus aureus ATCC 33592 (MRSA) and Listeria monocytogenes DSM 20600.

\section{Fraction Collection and Analysis}

Crude extracts were fractionated by high-performance liquid chromatography (HPLC) using a Dionex ICS 3000 instrument equipped with a Dionex Acclaim 120 C8 $3 \mu \mathrm{m} 2.1 \times 150 \mathrm{~mm}$ column (Thermo Fisher Scientific, Waltham, MA, United States). Eluent A consisted of $1 \%$ formic acid in water and eluent B consisted of $1 \%$ formic acid in acetonitrile, flowing at a rate of $250 \mu \mathrm{l} / \mathrm{min}$ at a column temperature of $35^{\circ} \mathrm{C}$. The elution profile was $20 \%$ B isocratic for $5 \mathrm{~min}$, then a gradient from 20 to $100 \% \mathrm{~B}$ in $55 \mathrm{~min}$, then $100 \% \mathrm{~B}$ isocratic for $15 \mathrm{~min}$, and then a return to the initial conditions in 1 min followed by re-equilibration for $14 \mathrm{~min}$. Fractions were collected during the first $75 \mathrm{~min}$. We injected $10 \mu \mathrm{l}$ of bioactive, filtered crude extract into the system for separation. Subsequently, the fractions were analyzed by highresolution electrospray ionization quadrupole time-of-flight mass spectrometry (QqTOF-ESI-HRMS) using a Bruker Daltonics instrument (Bremen, Germany) running oTOF Control v3.4 and Compass v1.7. The instrument was equipped with an orthogonal ESI source. Source parameters were adjusted as follows: capillary voltage $4.5 \mathrm{kV}$, end plate offset $500 \mathrm{~V}$, nebulizer gas $1.6 \mathrm{bar}$, dry gas $8 \mathrm{l} / \mathrm{min}$ with a dry temperature of $200^{\circ} \mathrm{C}$. Samples were screened in positive-ion mode. The mass spectrometer was coupled to a Dionex UltiMate 3000 HPLC system running under Chromeleon Express (Thermo Fisher Scientific). Both instruments were controlled using HyStar v3.2 SR 4.

\section{FLASH Chromatography}

Compounds were isolated following large-scale fermentation (5 1 volume). The crude methanol extract was treated multiple times with acetonitrile to precipitate any proteins. The precipitate was filtered and dissolved in water, then freeze-dried and dissolved in methanol at a concentration of $50 \mathrm{mg} / \mathrm{ml}$. The organic and aqueous phases $(50 \mathrm{mg} / \mathrm{ml})$ were again tested for bioactivity against Staphylococcus aureus. The bioactive extract was then mixed with an equal amount of Celite before drying in a rotary evaporator under reduced pressure. The Celite-sample mixture was used to prepare a pre-column. The samples were separated on a C18 reversed-phase column (Interchim PuriFlash C18-AQ $30 \mu \mathrm{M}$ F0120) using water as eluent $\mathrm{A}$ and acetonitrile as eluent B. The elution profile was $20 \% \mathrm{~B}$ isocratic for $8 \mathrm{~min}$, then a gradient from 20 to $100 \% \mathrm{~B}$ in $45 \mathrm{~min}$, then $100 \% \mathrm{~B}$ isocratic for $10 \mathrm{~min}$. Fractions were collected in a peak-dependent manner (i) at $210 \mathrm{~nm}$, (ii) at $254 \mathrm{~nm}$, and (iii) targeting peaks visualized using an evaporative light-scattering detector. The reaction tubes were combined in a logical order to reunite separated peak fractions and the solvents were evaporated. The remaining 
substances were then dissolved in methanol at $25 \mathrm{mg} / \mathrm{ml}$ and retested for antimicrobial activity using the growth inhibition assay described above.

\section{NMR Spectroscopy}

The purified antimicrobial compound was analyzed by ${ }^{1} \mathrm{H}$ and ${ }^{13} \mathrm{C}$ NMR spectroscopy, as well as correlation spectroscopy (COSY), heteronuclear single quantum coherence (HSQC) spectroscopy and heteronuclear multiple-bond correlation (HMBC) spectroscopy using a Bruker AV400 device. Chemical shifts were referenced to the methanol-d6 solvent residual peaks, $\delta H=3310$ ppm for ${ }^{1} \mathrm{H}$ and $\delta H=49,000$ ppm for ${ }^{13} \mathrm{C}$.

\section{Nematode Motility Assay}

Caenorhabditis elegans was grown on nematode growth medium (NGM) covered with a lawn of E. coli OP20 for 4 days at $20^{\circ} \mathrm{C}$. The worms were washed off the Petri dish into a 15 ml Falcon tube using a glass Pasteur pipette and M9 buffer. The nematode suspension was then centrifuged at $440 \mathrm{~g}$ for $2 \mathrm{~min}$ and the supernatant was removed. The pellet was washed again with M9 buffer and, after another step of centrifugation as described above, the content of the tube was reduced to $3.5 \mathrm{ml}$. To eliminate the worms and synchronize the suspension, we added $1.5 \mathrm{ml}$ of bleach mix $(0.5 \mathrm{ml} 5 \mathrm{M} \mathrm{NaOH}, 0.5 \mathrm{ml}$ $\mathrm{NaOCl}$, and $0.5 \mathrm{ml}$ water). The suspension was briefly mixed and shaken until the color of the solution changed from yellowish to clear (4-6 min). The tube was filled up to $15 \mathrm{ml}$ with M9 buffer and centrifuged for another $4 \mathrm{~min}$ at $2760 \mathrm{~g}$. The supernatant was quickly removed without touching the pellet until only $0.1 \mathrm{ml}$ remained, and $15 \mathrm{ml}$ of $\mathrm{M} 9$ buffer was added. In order to completely remove the bleach mix, the tube was carefully inverted and the pellet was rinsed three times with M9 buffer before centrifugation at $2760 \mathrm{~g}$ to remove all liquid. Finally, we added $10 \mathrm{ml}$ of M9 buffer and $10 \mu \mathrm{l}$ of cholesterol ( $5 \mathrm{mg} / \mathrm{ml}$ in $99 \%$ ethanol). Nematodes hatched overnight while the culture was shaking at room temperature. The nematodecontaining medium was centrifuged for $4 \mathrm{~min}$ at $1200 \mathrm{~g}$ to remove all liquid. After a final washing step with $15 \mathrm{ml}$ M9 buffer, the freshly hatched nematodes were centrifuged and the concentration was adjusted to $10 \mathrm{~L} 1$ nematodes per $100 \mu \mathrm{l}$ by diluting with NGM seeding medium. The latter was prepared by supplementing $10 \mathrm{ml} \mathrm{NGM}$ with $10 \mu \mathrm{l} 5 \mathrm{mg} / \mathrm{ml}$ cholesterol, $10 \mu \mathrm{l} 25 \mathrm{mg} / \mathrm{ml}$ carbenicillin and $50 \mu \mathrm{l}$ of an E. coli OP50 overnight culture.

The tests were carried out in triplicate in a 96-well plate with ivermectin (10 $\mathrm{gg} / \mathrm{ml}$ in DMSO) as the positive control. Serrawettin W2 was readily isolated from a culture of Serratia marcescens 2MH3-2 and purified in the course of a bioactivity-guided screening as described. A stock solution in DMSO was serially diluted from 256 to $2 \mu \mathrm{g} / \mathrm{ml}$. DMSO was used as the negative control. Nonmotile nematodes were counted under the microscope after $24 \mathrm{~h}$. To test for nematicidal effects, the incubated suspension was diluted 1:10 in M9 buffer and seeded on NGM agar plates containing E. coli OP50. The Petri dishes were checked for vital nematodes after 4 days of incubation at room temperature.

\section{RESULTS}

\section{Characterization of the $N$. vespilloides Gut Microbiome}

After the dissection process and microbial cultivation, we were able to pick more than 400 isolates from the gut of $N$. vespilloides. Nevertheless, no distinct differences between microbial cultivates from wild caught or bred beetles were observed. The obtained isolates were checked for contaminations and identified by colony PCR and Sanger sequencing (Eurofins, Germany). A sufficient read length of the $16 S$ rDNA gene was ensured by the assembly of the forward and reverse reads to a total of $\sim 1300 \mathrm{bp}$. The results were analyzed by BLASTn (NCBI), de-replicated and sorted according to the genus. The cultivated diversity is depicted in Figure 1A. We generated bacterial isolates from the Proteobacteria $(\sim 3 \%$ Alphaproteobacteria, $\sim 1 \%$ Betaproteobacteria and $\sim 68 \%$ Gammaproteobacteria), 3\% Flavobacteria, 20\% Bacilli and $\sim 5 \%$ Actinobacteria. Enterobacteriales was the most abundant order, to which $\sim 49 \%$ of all bacterial isolates belonged. Within the order Enterobacteriales, Serratia $(\sim 39 \%)$, Hafnia $(\sim 23 \%)$, Proteus $(\sim 16 \%)$ and Morganella $(\sim 11 \%)$ were the most prevalent genera (Figure 1B). Furthermore, the genera Pseudomonas (order: Pseudomonadales) and Stenotrophomonas (order: Xanthomonadales) were also strongly represented in the phylum Gammaproteobacteria. Other prevalent genera included Vagococcus and Carnobacterium as well as Enterococcus and Lactococcus (order: Lactobacillales). The genera listed above accounted for more than $70 \%$ of all cultivated isolates. In addition to these major genera, we also cultivated Enterobacter, Citrobacter, and Erwinia (order: Enterobacteriales) and Acinetobacter (order: Pseudomonadales). The order Flavobacteriales was solely represented by the genus Chryseobacterium, although the latter was less prevalent than the other genera. We were also able to cultivate Variovorax and Myroides as representatives of the order Burkholderiales, and Wohlfartiimonas, which has not yet been assigned to an order.

\section{Antimicrobial Activity of the Chemical Extracts}

Fermentations were established with several representative species. Cell suspensions were harvested on days 1, 6, and 9 for chemical extraction and analysis to ensure we did not miss the late induction of genes responsible for potential bioactive compounds under nutritional stress, such as carbon limitation. After chemical extraction and concentration, the crude extracts were screened against selected human-pathogenic Gram-positive and Gram-negative bacterial strains, as well as a clinically relevant strain of Candida albicans to identify antimicrobial activity. More than 800 crude extracts were screened for growth inhibition of test strains, revealing a broad spectrum of bioactivities. Almost none of our ethyl acetate extracts showed inhibition, so the effects of our methanol extracts were prioritized and a selection is summarized in Figure 2. A large set of families within the orders of Micrococcales, Enterobacteriales, 

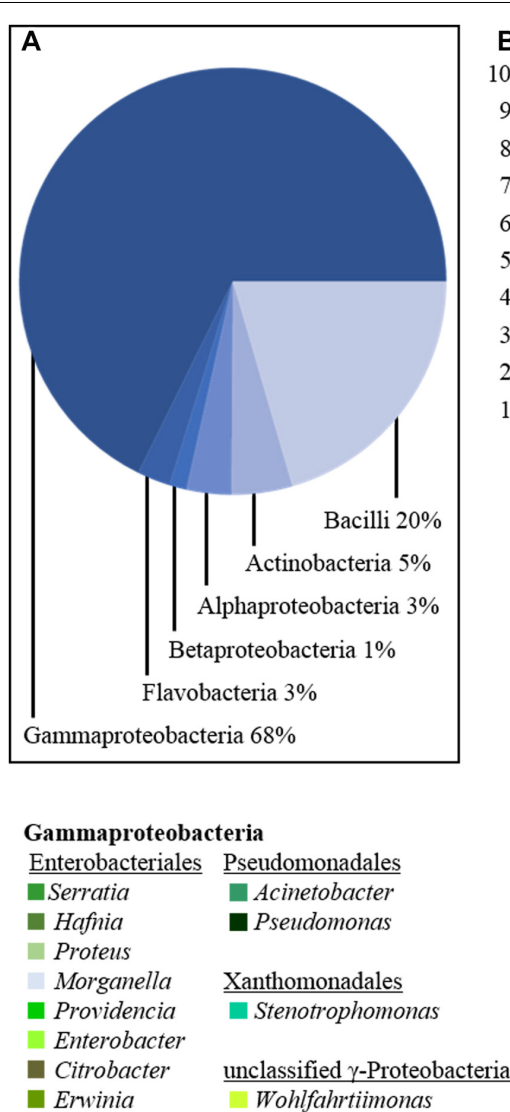

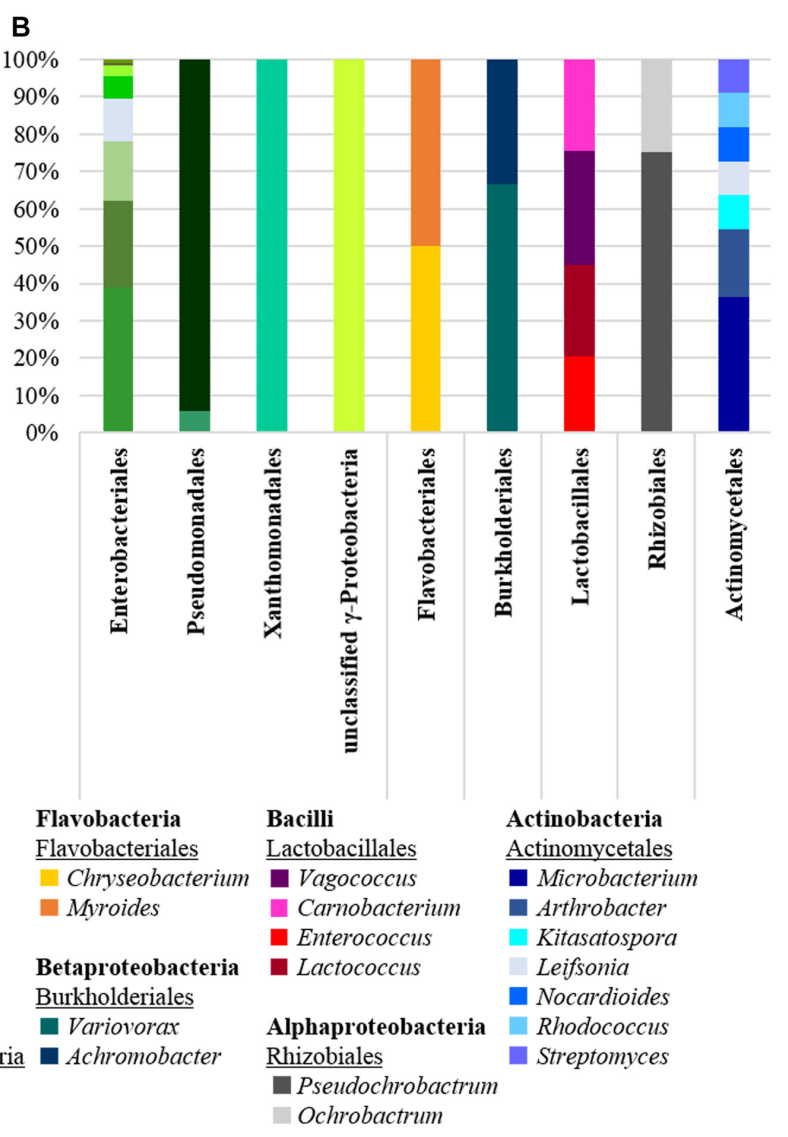

FIGURE 1 | Diversity of cultivated bacteria isolated from dissected beetle guts. (A) The class of Gammaproteobacteria is the most abundant one isolated with $68 \%$ of all cultures obtained, followed by Bacilli (20\%) and Actinobacteria (5\%). Further, also Flavobacteria (3\%), Alphaproteo- (3\%), and Betaproteobacteria (1\%) were cultivated. (B) The class of Gammaproteobacteria is represented by the orders of Enterobacteriales, Pseudomonadales, Xanthomonadales and a single unclassified genus (Wohlfartiimonas). Enterobacteriales was the order with the highest species diversity isolated. The second prominent phylum of Bacilli was represented by only four genera of the order Lactobacillales. The phylum Actinobacteria was represented by the order of Actinomycetales. Compared to the number of isolates, the species diversity within this order was surprisingly high, although other more abundant phyla appeared to be much less diverse.

and Lactobacillales with different levels of antimicrobial activity against our screening strains were observed. The broadest antimicrobial activity was found in the extracts of strains 89 , 38, 42 belonging to the genus Enterococcus and the strains FF6$\mathrm{H}_{2} \mathrm{O}$ and 56 belonging to the genus Serratia. Crude extracts of these two strains displayed strong inhibition of all strains tested. The extracts of strains 89, 38, 42 showed potent antimicrobial effects against $E$. coli as well as $P$. aeruginosa and Staphylococcus aureus, with more than $90 \%$ growth inhibition and at least $60 \%$ inhibition of C. albicans. The crude extracts of the genera Pseudomonas showed the highest bioactivity. Almost $20 \%$ of the observed bioactivities were derived from Pseudomonas, followed by Enterobacter and Serratia, both accounting for $\sim 12 \%$ of the total activity. Overall, $71 \%$ of all methanol crude extracts were capable of inhibiting of $M$. smegmatis. In contrast, selective inhibition of $C$. albicans has only been rarely observed. One of those examples is the isolate Wild Mid 14 (Figure 2), obtained from the midgut of a wild living beetle. Only $11 \%$ of all extracts displayed antimicrobial activity against Gram-negative bacteria. Thereof, the genera Enterococcus and
Serratia yielded extracts that exhibited pronounced anti-Gramnegative activity.

As mentioned above, our microbiome cultivation included a relatively large number of different Serratia species, some of which caused the complete inhibition of our screening strains. The broadest inhibitory effects were observed with the crude methanol extract obtained after 9 days of cultivation of the strains FF6- $\mathrm{H}_{2} \mathrm{O}$ and 56, both identified as Serratia plymuthica. Several more antimicrobial effects could be observed with the crude extracts derived from additional Serratia isolates. The most frequently inhibited pathogen was $M$. smegmatis, because all tested Serratia-derived extracts displayed bioactivity. However, inhibitory effects against $C$. albicans were rare. To correlate bioactivity and phylogeny of the Serratia species obtained, the observed growth inhibitions were plotted to a maximumlikelihood phylogenetic tree (Figure 3). Partial $16 S$ sequences as well as reference $16 S$ sequences of $S$. grimesii, S. proteamaculans, $S$ ficaria, $S$. vespertiliones, $S$. entomophilia, S. symbiotica, S. plymuthica, S. liquefaciens, S. glossinae, S. fonticola, S. aquatilis, S. rubidaea, S. ureilytica and $S$. marcescens (all NCBI type 


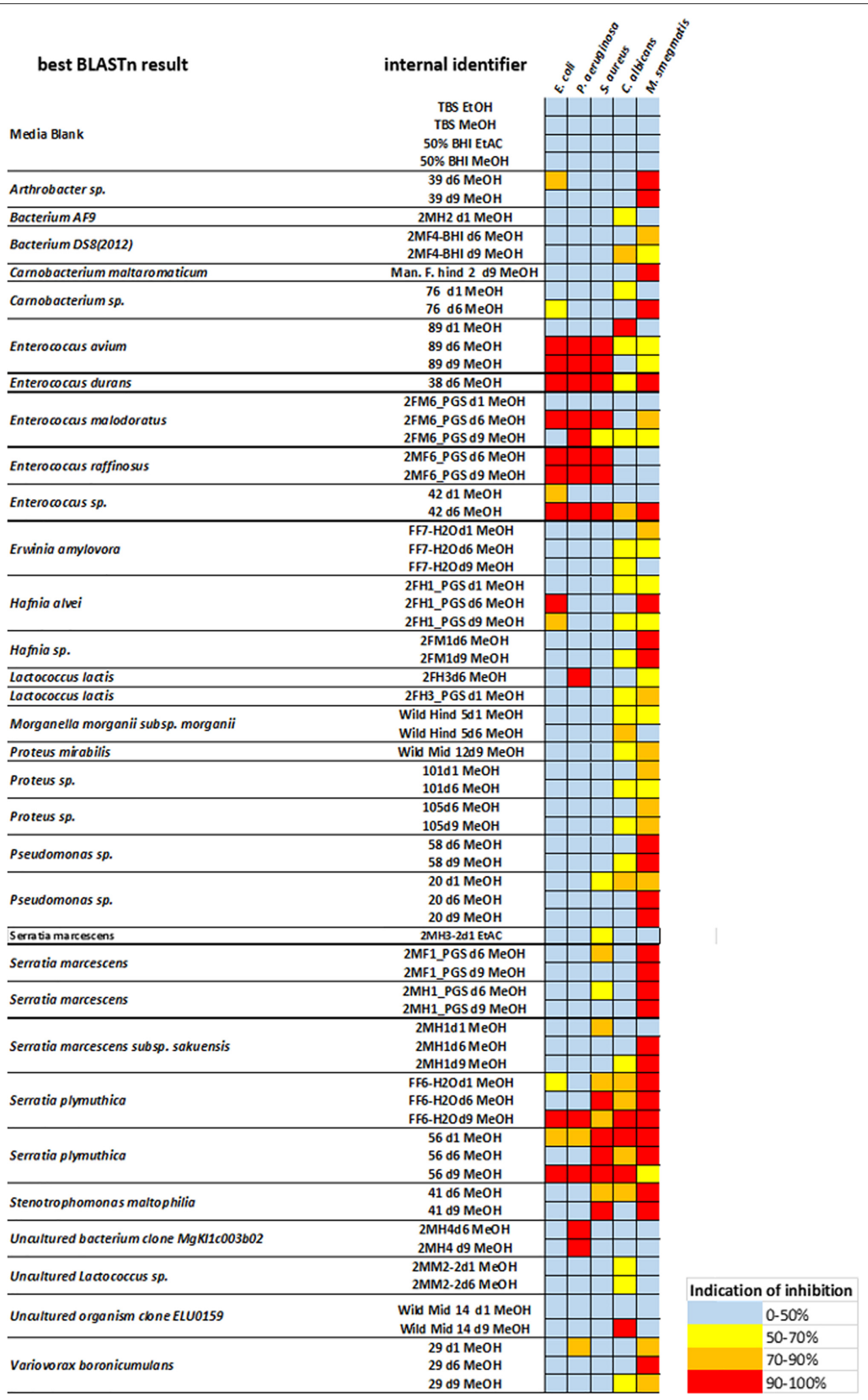

FIGURE 2 | Antimicrobial activity of selected N. vespilloides gut bacteria against five representative bacterial pathogens. The color key indicates the percentage of growth inhibition of Escherichia coli ATCC 25922, Pseudomonas aeruginosa ATCC 27853, Staphylococcus aureus ATCC 25923, and Candida albicans. Single inhibition effects of Mycobacterium smegmatis are not displayed here because of the high amount of positive results. Column 1 displays the best BLAST result of the $16 \mathrm{~S}$ analysis, column 2 contains the internal strain names, including the duration of fermentation (e.g., $\mathrm{d} 6=6$ days of fermentation) and the solvent used for extraction. On the right side, a heat map displays the range of antimicrobial effects observed. 


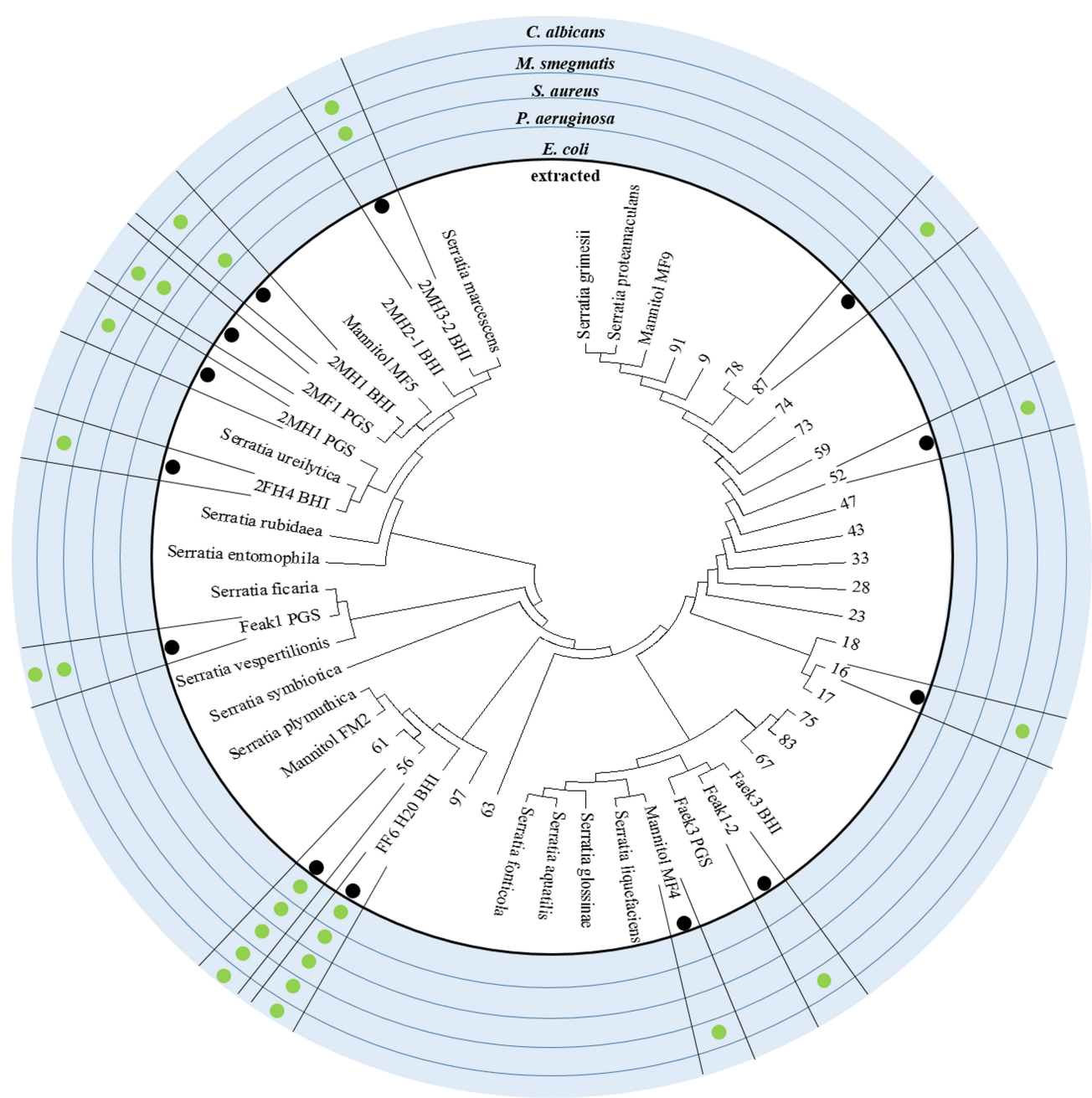

FIGURE 3 | Phylogeny of the Serratia isolates and their antibiotic active crude extracts. The alignment of 16S rDNA sequences with Serratia reference strains from the NCBI database is shown here as a maximum likelihood phylogenetic tree. The outer circles indicate if the isolated strain was tested for antimicrobial effect and against which pathogen an effect could be reported. Strains such as $\mathrm{FF} 6-\mathrm{H}_{2} \mathrm{O}$ and 56 display outstanding, broad spectrum antimicrobial activity and group around the reference strain S. plymuthica.

strains) were used to generate this phylogenetic tree. The strains FF6- $\mathrm{H}_{2} \mathrm{O}$ and 56, with their broad antimicrobial activity, were identified as $S$. plymuthica. Both strains belong to the clade of the reference $S$. plymuthica strain and we are currently working on the identification of the active compounds in these extracts. Several further isolates showed diverse antimicrobial effects. For example, the isolate Faek1 derived from the anal secretions of $N$. vespilloides displayed antifungal activity against $C$. albicans.

Isolate 2MH3-2 (S. marcescens) was chosen for further investigation due to its remarkably strong inhibition of Staphylococcus aureus ATCC 25923 (MSSA). To determine the structure of the antimicrobial component, the bioactive crude extract was fractionated by HPLC for bioactivity testing, revealing a single fraction that completely inhibited this pathogen (Figure 4A). Analysis of this fraction revealed one dominant peak in the base peak chromatogram (Figure 4B), which corresponded to $m / z 732.4464\left[(\mathrm{M}+\mathrm{H})^{+}\right]$as determined by QqTOF-ESI-HRMS (Figure 4C). Large-scale fermentation (51) was therefore carried out to isolate the active compound. Initial fractionation by flash chromatography and subsequent bioactivity-guided purification of the inhibitory fraction by HPLC led to the isolation of the target molecule. NMR spectroscopy, focusing on the ${ }^{1} \mathrm{H}$ and HSQC spectra, revealed five $\alpha-H$ signals at $\delta_{H}$, namely $4.61($ Phe- $\alpha H), 4.52($ Leu- $\alpha H)$, 4.37 (Ser- $\alpha \mathrm{H}), 4.18$ (Ile- $\alpha \mathrm{H})$ and 4.11 (Thr- $\alpha \mathrm{H})$, thus indicating the presence of a pentapeptide. This result was confirmed by HMBC spectroscopy. The aliphatic protons resonated between $\delta_{\mathrm{H}} 2.61$ and 1.16, suggesting the presence of a lipopeptide. The prominent pseudomolecular ion $m / z 732.4464\left([\mathrm{M}+\mathrm{H}]^{+}\right)$in the QqTOF-ESI-HRMS spectrum agreed with the calculated $m / z$ of 732.4469 ( $\Delta m / z$ 0.0005). The molecular formula $\mathrm{C}_{38} \mathrm{H}_{61} \mathrm{~N}_{5} \mathrm{O}_{9}$ was therefore assigned. Combining the QqTOF-ESI-HRMS and NMR data, the compound was unambiguously identified as 


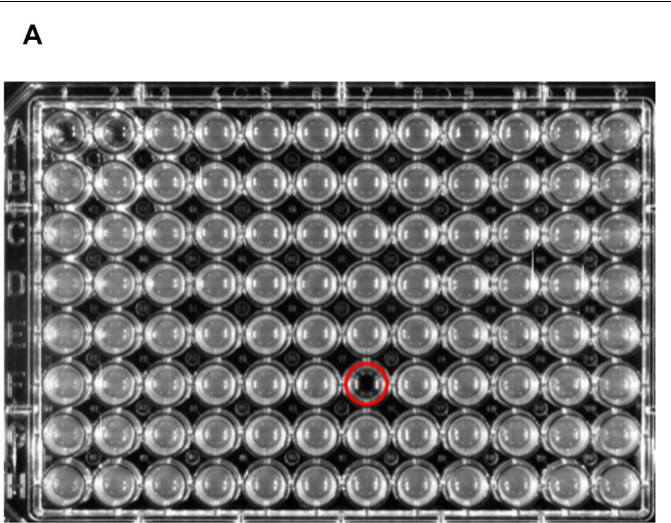

D

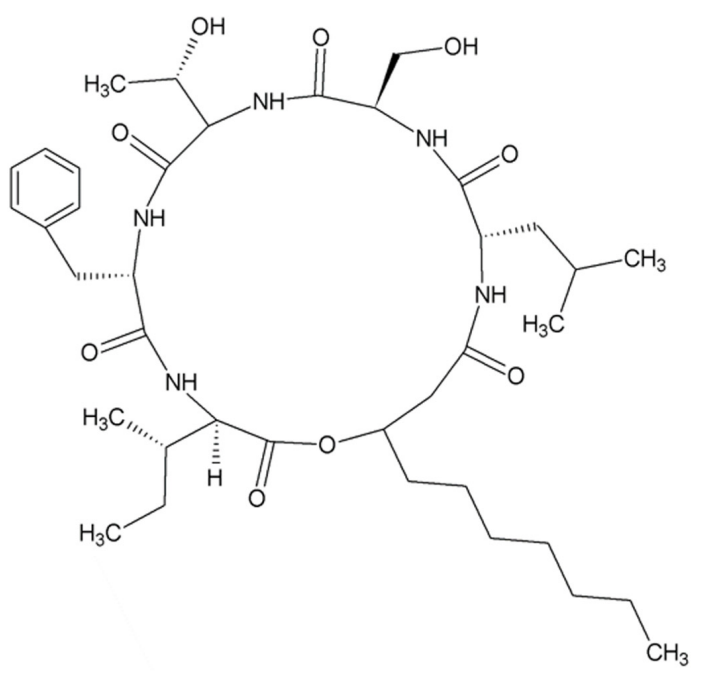

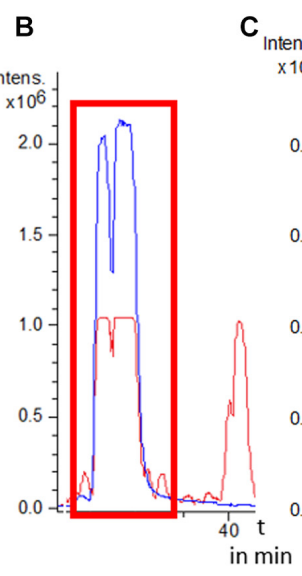

calc: $731.4469 \mathrm{~m} / \mathrm{z}$

$\mathrm{C}_{38} \mathrm{H}_{61} \mathrm{~N}_{5} \mathrm{O}_{9}$

FIGURE 4 | Identification of serrawettin W2. (A) HPLC fraction collection and biological tests for antimicrobial activity against Staphylococcus aureus resulted in the identification of a single active fraction. (B,C) LC-MS analysis revealed a major peak in the base peak chromatogram (red line) of the crude extract as the active fraction, $m / z=732.4464[\mathrm{M}+\mathrm{H}]^{+}$. The extracted ion chromatogram (blue line) $\mathrm{m} / \mathrm{z} 732.43-732.46$ highlights the peak segment with antimicrobial activity. (D) Structure of serrawettin $\mathrm{W} 2\left(\mathrm{C}_{38} \mathrm{H}_{61} \mathrm{~N}_{5} \mathrm{O}_{9}\right)$, a cyclodepsipeptide with known antimicrobial activity and a $\mathrm{m} / z$ of $731.4469[\mathrm{M}]^{+}$.

TABLE 1 | Minimal inhibitory concentrations (MICs) of pure serrawettin W2 against representative Gram-positive and Gram-negative bacteria.

\begin{tabular}{lllc}
\hline Test strain & Accession number & Genotype & MIC $(\boldsymbol{\mu} \mathbf{g} / \mathbf{m l})$ \\
\hline E. coli & ATCC 25922 & wild type & $>128$ \\
E. coli & ATCC 25922 & DtolC mutant & $>128$ \\
B. subtilis & DSM 10 & wild type & 4 \\
S. aureus & ATCC 25923 & MSSA & 4 \\
S. aureus & ATCC 33592 & MRSA & 4 \\
L. monocytogenes & DSM 20600 & wild type & 4 \\
\hline
\end{tabular}

serrawettin W2, a cyclic pentadepsipeptide (Figure 4D) that has previously been isolated from other strains of Serratia marcescens (Matsuyama et al., 1992). The purified compound showed no antibiotic activity against E. coli ATCC 25922 or the E. coli $\Delta$ tolC mutant. Notably, the MICs against the four Gram-positive test strains were in each case $4 \mu \mathrm{g} / \mathrm{ml}$ (Table $\mathbf{1}$ ).
Serrawettin W2 was previously identified as a nematode repellent (Pradel et al., 2007) but we hypothesized that it may also act as a nematicide, based on the need to suppress competing and parasitic nematodes transmitted to carcasses attended by $N$. vespilloides. We therefore conducted livedead assays by exposing the model nematode $C$. elegans to a broad range of serrawettin W2 concentrations in technical triplicates. We observed $100 \%$ immotility at a concentration of $128 \mu \mathrm{g} / \mathrm{ml}$, allowing us to extrapolate an $\mathrm{ED}_{50}$ value of $25.27 \mu \mathrm{g} / \mathrm{ml}$ based on an exponential trend line (Figure 5). Our results showed that serrawettin W2 is nematostatic at $128 \mu \mathrm{g} / \mathrm{ml}$, but there were also fewer survivors compared to the control treatment, indicating the potential for nematicidal activity. Accordingly, when we seeded nematodes on NGM agar covered with a lawn of $E$. coli and exposed them to $256 \mu \mathrm{g} / \mathrm{ml}$ serrawettin W2, we observed a complete absence of feeding tracks (indicating live worms), confirming that the antibiotic is nematicidal at this higher concentration. We 


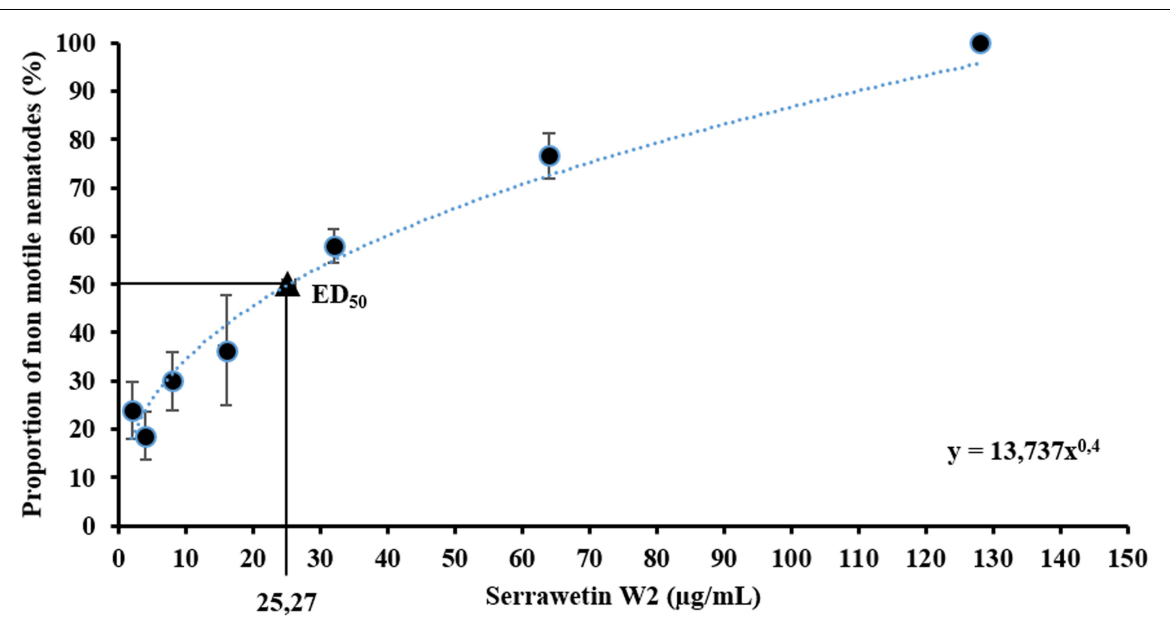

FIGURE $\mathbf{5}$ | Nematode motility assay with serrawetin W2. Non-motile nematodes were counted under the microscope, and the abundance of non-motile nematodes was calculated. By displaying the experimental data (average and standard error), a resulting effective dose (ED 50 ) was extrapolated.

therefore consider serrawettin W2 primarily as a nematostatic compound but as a nematicide at concentrations greater than $128 \mu \mathrm{g} / \mathrm{ml}$.

\section{DISCUSSION}

The larval and adult guts of the burying beetle $N$. vespilloides harbor a diverse microbial community including the Firmicutes, Proteobacteria and ascomycetous yeasts of the genus Yarrowia. Certain members of the microbiome are transmitted to the carcass via anal secretions (Vogel et al., 2017). This seeding strategy was recently proposed to facilitate the suppression of microbial competitors based on evidence of metabolic cooperation between the host and its microbiome for digestion, detoxification and defense (Duarte et al., 2018). Shukla et al. (2018b) recently corroborated this hypothesis and underlined the pivotal role of symbionts representing the genus Serratia in the preservation of carcasses prepared by burying beetles.

Using a distinct experimental approach by cultivating and characterizing bacterial strains isolated from the gut of $N$. vespilloides, we confirmed in this study the composition of its gut microbiota. Inspired by the hypothesis that the core microbiome of $N$. vespilloides contains bacteria, which defend the host against environmental microbes associated with the carcass and taken up with the diet, we screened the supernatants of gut bacteria cultures against a set of human pathogens to identify antimicrobial compounds. Subsequently, we observed a large variety of different antimicrobial effects, partly summarized in Figure 1. In this context, we observed strong activity against $S$. aureus in supernatants from an isolate identified as $S$. marcescens. Subsequent characterization of the antimicrobial compound resulted in its identification as serrawettin W2. The latter molecule consists of five amino acids and an additional fatty acid residue (Matsuyama et al., 1992). This cyclic lipodepsipentapeptide displayed potent activity against the Gram-positive bacterium S. aureus ATCC 33592 (MRSA) in our growth inhibition assays. Shukla et al. (2018a) revealed the presence of staphylococci on untended carcasses whereas carcasses prepared by burying beetles are devoid of these bacteria. This agrees with our finding that serrawettin W2 has potent inhibitory activity against Staphylococcus aureus. The most prominent group of Serratia antibiotics are undoubtedly the red pyrrole alkaloid prodigiosin and its derivatives (Williams, 1973; Fürstner, 2003; Lapenda et al., 2015). Despite this, none of the Serratia isolates investigated in this study formed a red pigment. Apart from these pigments, carbapenem antibiotics have been reported that are produced by Serratia species (Bycroft et al., 1987). A Serratia sp. was reported as the producer of six cyclodepsipeptides with distinctive anti-mycobacterial activity. Notably, serratomolides A-F displayed antibacterial activity against Mycobacterium diernhoferi and additional rapidly growing mycobacteria, but no inhibition of other human pathogenic Gram-positive and Gram-negative bacteria was observed (Dwivedi et al., 2008).

Furthermore, pure serrawettin W2 showed strong nematostatic effects against the model organism C. elegans. This indicates that serrawettin W2 is not only a nematode repellant as previously reported (Pradel et al., 2007) but also a potent nematostatic molecule. The ability to deter nematodes is of particular importance to burying beetles, which are associated with parasitic nematodes, some of which are exclusively associated with $N$. vespilloides (Richter, 1993). The nematode dauer juveniles use the beetles' gut as a vehicle to transfer them to carrion, where they feed and reproduce and thus achieve trans-generational transmission from parent to larvae through the carcass surface and direct parental interactions. The dauer juveniles of the offspring generation migrate to the genitalia and 
the gut of the hatching beetles to use them for transfer to the next carcass (Richter, 1993). Transcriptomic analysis of the feeding cavity of beetle-prepared carcasses (where larvae reside and feed) indicates that the nematodes are not only prevalent along with $N$. vespilloides, but are also metabolically active, competing for carcass nutrients with the beetle larvae (Vogel et al., 2017). Recently, another phoretic nematode associated with $N$. vespilloides (Rhabditoides regina) was described as a parasite because it negatively affected the beetle's brood size, larval survival and larval mass (Wang and Rozen, 2019). Because Rhabditoides nematodes can influence carcass health, larval survival and beetle fitness, we postulate that microbes in the $N$. vespilloides gut producing serrawettin W2 may help the beetles to suppress nematode populations in the gut as well as on the carcass surface.

The discovery of antibiotic-producing bacteria in the gut of $N$. vespilloides indicates the presence of microbial symbionts contributing to the control of bacteria and nematodes, which could harm the eggs laid near by the carcass (Jacobs et al., 2014) and compete with the larval offspring to exploit the carcass as a highly nutritious but ephemeral food source. The results presented here fit well with the proposed strategies for managing rival bacterial communities in $N$. vespilloides (Duarte et al., 2018).

Our study suggests that the strategy of outsourcing defense against pathogens and parasites to bacterial symbionts is more widespread in beetles than previously thought (Dose et al., 2018; Flórez et al., 2018; Schmidtberg et al., 2019). The symbiotic microbiome of beetles therefore offers an untapped resource for the discovery of novel natural products with activity against human pathogens and parasites.

\section{REFERENCES}

Bycroft, B. W., Maslen, C., Box, S. J., Brown, A. G., and Tyler, J. W. (1987). The isolation and characterisation of $(3 R, 5 R)$ - and $(3 S, 5 R)$-carbapenam3-carboxylic acid from Serratia and Erwinia species and their putative biosynthetic role. J. Chem. Soc. Chem. Commun. 1623-1625. doi: 10.1039/ C39870001623

Dardic, D., Lauro, G., Bifulco, G., Laboudie, P., Sakhaii, P., Bauer, A., et al (2017). Svetamycins A-G, unusual piperazic acid-containing peptides from Streptomyces sp. J. Organ. Chem. 82, 6032-6043. doi: 10.1021/acs.joc.7b 00228

Degenkolb, T., Düring, R.-A., and Vilcinskas, A. (2011). Secondary metabolites released by the burying beetle Nicrophorus vespilloides: chemical analyses and possible ecological functions. J. Chem. Ecol. 37, 724-735. doi: 10.1007/s10886011-9978-4

Dose, B., Niehs, S. P., Scherlach, K., Flórez, L. V., Kaltenpoth, M., and Hertweck, C. (2018). Unexpected bacterial origin of the antibiotic icosalide: two-tailed depsipeptide assembly in multifarious Burkholderia symbionts. ACS Chem. Biol. 13, 2414-2420. doi: 10.1021/acschembio.8b00600

Duarte, A., Welch, M., Swannack, C., Wagner, J., and Kilner, R. M. (2018). Strategies for managing rival bacterial communities: lessons from burying beetles. J. Anim. Ecol. 87, 414-427. doi: 10.1111/1365-2656.12725

Dwivedi, D., Jansen, R., Molinari, G., Nimtz, M., Johri, B. N., and Wray, V. (2008). Antimycobacterial serratamolides and diacyl peptoglucosamine derivatives from Serratia sp. J. Nat. Prod. 71, 637-641. doi: 10.1021/np7007126

\section{DATA AVAILABILITY}

Sequences of Serratia isolates are deposited in GenBank (MK386907-MK386931 and MK391500-MK391513).

\section{AUTHOR CONTRIBUTIONS}

HV, TS, and AV designed the experiments. PH performed the microbial and molecular work, processed the samples, analyzed the data, and prepared the manuscript. YL isolated the compound and performed the structure elucidation. TD conducted the LC-MS experiments, analyzed the data, and prepared the manuscript.

\section{FUNDING}

This project was funded by the Fraunhofer Society and Max Planck Society via the collaborative project AIM-Biotech (Application of Insect-associated Microbes in industrial biotechnology) and the HMWK via the LOEWE Center for Insect Biotechnology and Bioresources. Parts of the work in the lab of TS were funded by the German Center for Infection Research (DZIF).

\section{ACKNOWLEDGMENTS}

The authors thank Benedikt Leis (Fraunhofer IME BR, Giessen) for performing the antibacterial and nematicidal testing. They are also indebted to Richard M. Twyman (Twyman Research Management Ltd., York, United Kingdom) who carefully edited a preliminary version of the manuscript.

Engl, T., Kroiss, J., Kai, M., Nechitaylo, T. Y., Svatoš, A., and Kaltenpoth, M. (2018). Evolutionary stability of antibiotic protection in a defensive symbiosis. Proc. Natl. Acad. Sci. U.S.A. 115, E2020-E2029. doi: 10.1073/pnas.1719797115

Flórez, L. V., Scherlach, K., Gaube, P., Ross, C., Sitte, E., Hermes, C., et al. (2017). Antibiotic-producing symbionts dynamically transition between plant pathogenicity and insect-defensive mutualism. Nat. Commun. 8:15172. doi: 10.1038/ncomms 15172

Flórez, L. V., Scherlach, K., Miller, I. J., Rodrigues, A., Kwan, J. C., Hertweck, C., et al. (2018). An antifungal polyketide associated with horizontally acquired genes supports symbiont-mediated defense in Lagria villosa beetles. Nat. Commun. 9:2478. doi: 10.1038/s41467-018-04955-6

Fürstner, A. (2003). Chemistry and biology of roseophilin and the prodigiosin alkaloids: a survey of the last 2500 years. Angew. Chem. Int. Ed. 42, 3582-3603. doi: 10.1002/anie.200300582

Jacobs, C. G. C., Steiger, S., Heckel, D. G., Wielsch, N., Vilcinskas, A., and Vogel, H. (2016). Sex, offspring and carcass determine antimicrobial peptide expression in the burying beetle. Sci. Rep. 6:25409. doi: 10.1038/srep 25409

Jacobs, C. G. C., Wang, Y., Vogel, H., Vilcinskas, A., van der Zee, M., and Rozen, D. E. (2014). Egg survival is reduced by grave-soil microbes in the carrion beetle, Nicrophorus vespilloides. BMC Evol. Biol. 14:208. doi: 10.1186/s12862014-0208-x

Kim, D., Hofstaedter, C. E., Zhao, C., Mattei, L., Tanes, C., Clarke, E., et al. (2017). Optimizing methods and dodging pitfalls in microbiome research. Microbiome 5:52. doi: 10.1186/s40168-017-0267-5 
Kroiss, J., Kaltenpoth, M., Schneider, B., Schwinger, M. G., Hertweck, C., Maddula, R. K., et al. (2010). Symbiotic streptomycetes provide antibiotic combination prophylaxis for wasp offspring. Nat. Chem. Biol. 6, 261-263. doi: 10.1038/ nchembio. 331

Lane, D. J. (1991). “16S/23S rRNA sequencing”, in Nucleic Acid Techniques in Bacterial Systematics (Modern Microbiological Methods), eds E. Stackebrandt and M. Goodfellow (New York, NY: John Wiley and Sons), 115-175.

Lapenda, J. C., Silva, P. A., Vicalvi, M. C., Sena, K. X., and Nascimento, S. C. (2015). Antimicrobial activity of prodigiosin isolated from Serratia marcescens UFPEDA 398. World J. Microbiol. Biotechnol. 31, 399-406. doi: 10.1007/s11274014-1793-y

Lasken, R. S., and McLean, J. S. (2014). Recent advances in genomic DNA sequencing of microbial species from single cells. Nat. Rev. Genet. 15, 577-584. doi: $10.1038 / \operatorname{nrg} 3785$

Matsuyama, T., Kaneda, K., Nakagawa, Y., Isa, K., Harahotta, H., and Yano, I. (1992). A novel extracellular cyclic lipopeptide which promotes flagellumdependent and flagellum-independent spreading growth of Serratia marcescens. J. Bacteriol. 174, 1769-1776. doi: 10.1128/jb.174.6.1769-1776.1992

Miller, C. V. L., and Cotter, S. C. (2018). Resistance and tolerance: the role of nutrients on pathogen dynamics and infection outcomes in an insect host. J. Anim. Ecol. 87, 500-510. doi: 10.1111/1365-2656

Pradel, E., Zhang, Y., Pujol, N., Matsuyama, T., Bargmann, C. I., and Ewbank, J. J. (2007). Detection and avoidance of a natural product from the pathogenic bacterium Serratia marcescens by Caenorhabditis elegans. Proc. Natl. Acad. Sci. U.S.A. 104, 2295-2300. doi: 10.1073/pnas.0610281104

Pukowski, E. (1933). Ökologische Untersuchungen an Necrophorus. Z. Morphol. Ökol. Tiere 27, 518-586. doi: 10.1007/bf00403155

Richter, S. (1993). Phoretic association between the dauerjuveniles of Rhabditis stammeri (Rhabditidae) and life-history stages of the burying beetle Nicrophorus vespilloides (Coleoptera, Silphidae). Nematologica 39, 346-355. doi: 10.1163/ $187529293 \times 00295$

Rossiter, S. E., Fletcher, M. H., and Wuest, W. M. (2017). Natural products as platforms to overcome antibiotic resistance. Chem. Rev. 117, 12415-12474. doi: 10.1021/acs.chemrev.7b00283

Schmidtberg, H., Shukla, S. P., Halitschke, R., Vogel, H., and Vilcinskas, A. (2019). Symbiont-mediated chemical defence in the invasive ladybird Harmonia axyridis. Ecol. Evol. 9, 1715-1729. doi: 10.1002/ece3.48

Shukla, S. P., Plata, C., Reichelt, M., Steiger, S., Heckel, D. G., Kaltenpoth, M., et al. (2018a). Microbiome-assisted carrion preservation aids larval development in a burying beetle. Proc. Natl. Acad. Sci. U.S.A. 115, 11274-11279. doi: 10.1073/ pnas. 1812808115
Shukla, S. P., Vogel, H., Heckel, D. G., Vilcinskas, A., and Kaltenpoth, M. (2018b). Burying beetles regulate the microbiome of carcasses and use it to transmit a core microbiota to their offspring. Mol. Ecol. 27, 1980-1991. doi: 10.1111/mec. 14269

Stern, S., Chorzelski, S., Franken, L., Voller, S., Rentmeister, H., and Grosch, B. (2017). Breaking Through the Wall - A Call for Concerted Action on Antibiotics Research and Development. Follow-Up Report for the German Guard Initiative. Boston, MA: The Boston Consulting Group.

Vogel, H., Badapanda, C., and Vilcinskas, A. (2011). Identification of immunityrelated genes in the burying beetle Nicrophorus vespilloides by suppression subtractive hybridization. Insect Mol. Biol. 20, 787-800. doi: 10.1111/j.13652583.2011.01109.x

Vogel, H., Shukla, S. P., Engl, T., Weiss, B., Fischer, R., Steiger, S., et al. (2017). The digestive and defensive basis of carcass utilization by the burying beetle and its microbiota. Nat. Commun. 8:15186. doi: 10.1038/ncomms 15186

Wang, Y., and Rozen, D. E. (2018). Gut microbiota in the burying beetle, Nicrophorus vespilloides, provide colonization resistance against larval bacterial pathogens. Ecol. Evol. 8, 1646-1654. doi: 10.1002/ece3.3589

Wang, Y., and Rozen, D. E. (2019). Fitness costs of phoretic nematodes in the burying beetle, Nicrophorus vespilloides. Ecol. Evol. 9, 26-35. doi: 10.1002/ece3. 4570

Williams, R. P. (1973). Biosynthesis of prodigiosin, a secondary metabolite of Serratia marcescens. Appl. Microbiol. 25, 396-402.

Wilson, M. R., Zha, L., and Balskus, E. P. (2017). Natural product discovery from the human microbiome. J. Biol. Chem. 292, 8546-8552. doi: 10.1074/jbc.R116. 762906

Wright, G. D. (2017). Opportunities for natural products in $21^{\text {st }}$ century antibiotic discovery. Nat. Prod. Rep. 34, 694-701. doi: 10.1039/c7np00019g

Conflict of Interest Statement: The authors declare that the research was conducted in the absence of any commercial or financial relationships that could be construed as a potential conflict of interest.

Copyright (c) 2019 Heise, Liu, Degenkolb, Vogel, Schäberle and Vilcinskas. This is an open-access article distributed under the terms of the Creative Commons Attribution License (CC BY). The use, distribution or reproduction in other forums is permitted, provided the original author(s) and the copyright owner(s) are credited and that the original publication in this journal is cited, in accordance with accepted academic practice. No use, distribution or reproduction is permitted which does not comply with these terms. 